\title{
Isolation and characterization of cDNA clones encoding polypeptides related to a Dictyostelium discoideum cyclic AMP binding protein
}

\author{
Gerard Bain, $\dagger$ Caroline E. Grant $\ddagger$ and Adrian Tsang* \\ Department of Biology, McGill University, Montreal, Quebec, Canada H3A IBI
}

(Received 12 July 1990; revised 17 October 1990; accepted 9 November 1990)

\begin{abstract}
By screening a cDNA library with a cDNA encoding the Dictyostelium discoideum cAMP-binding protein CABP1, under conditions of reduced stringency, we have isolated clones which code for two closely related molecules. Hybrid selection experiments indicated that these cDNAs encoded polypeptides with molecular masses of 34 (p34) and 31 (p31) kDa, both of which were recognized by anti-CABP1 monoclonal antibodies. Sequence analysis revealed that the clones were identical except for the presence of a 102 nucleotide segment inserted in-frame in the p34 cDNAs, just downstream of the translation initiation codon. DNA blot analysis suggested that p34 and p31 were encoded by the same gene. This hypothesis was strongly supported by the observation that both polypeptides were generated when a single cDNA was expressed under the control of the actin 15 promoter in $D$. discoideum cells. RNA blot analysis indicated that the cDNAs were complementary to three developmentally regulated transcripts of sizes $1.15 \mathrm{~kb}, 1.25 \mathrm{~kb}$ and $1.4 \mathrm{~kb}$. Comparison of the derived amino acid sequences of p34 and p31 with those of the two subunits of CABP1 indicated that these polypeptides were very closely related, and that the corresponding genes probably arose by duplication followed by sequence divergence. Finally, the carboxy termini of these four polypeptides demonstrated $50 \%$ similarity to two polypeptides encoded by a bacterial plasmid which confers resistance to tellurium anions.
\end{abstract}

\section{Introduction}

The cellular slime mould Dictyostelium discoideum grows vegetatively as single-celled amoebae. Starvation triggers a process of development and differentiation, which ultimately leads to the formation of a multicellular fruiting body composed of two distinct cell types, spores and stalk cells (see Loomis, 1982, for a review). The molecule cAMP plays a pivotal role in the developmental cycle of $\mathrm{D}$. discoideum. It acts as a chemoattractant to direct the aggregation of individual amoebae after development has begun, regulates the differentiation of both spores and stalk cells, and controls the expression of many developmentally regulated genes (reviewed in Gerisch, 1987). While most of these effects are mediated

\footnotetext{
$\dagger$ Present address: Washington University School of Medicine, Department of Anatomy and Neurobiology, 660 South Euclid, St Louis, MO 63110, USA.

$\ddagger$ Present address: Cancer Research Labs, Botterell Hall, Queen’s University, Kingston, Ontario, Canada.

The nucleotide sequence data reported in this paper have been submitted to GenBank and have been assigned the accession numbers M36174 and M36175.
}

by the interaction between extracellular cAMP and a cell-surface cAMP receptor (reviewed in Firtel et al., 1989), it also appears that intracellular cAMP plays an important role in some of these processes (Kimmel, 1987; Kay, 1989; Riley et al., 1989).

In an attempt to identify possible pathways which could mediate the effects of intracellular cAMP on the development of $D$. discoideum, we have isolated and characterized a novel cAMP binding protein, CABP1 (Tsang \& Tasaka, 1986). This protein consists of two polypeptide subunits, CABP1A and CABP1B. Western blot analysis using anti-CABP1 monoclonal antibodies indicates that there are several CABP1-related polypeptides present in developing cells (Kay et al., 1987; Tsang et al. 1988).

Two of the polypeptides which crossreact with the anti-CABP1 antibodies are of particular interest because of their developmental regulation and intracellular localization. These molecules, which have apparent molecular masses of $34 \mathrm{kDa}(\mathrm{p} 34)$ and $31 \mathrm{kDa}(\mathrm{p} 31)$, are not detected in vegetatively growing cells, but appear early in development (Kay et al., 1987). They are localized primarily in the nucleus (Kay et al., 1987). In this paper, we describe the molecular cloning and 
characterization of cDNAs encoding p34 and p31. We show that these two polypeptides are very closely related to the two subunits of CABP1 and provide evidence that they are the products of a single gene.

\section{Methods}

Isolation of cDNA clones. A cDNA library was constructed according to standard procedures (Maniatis et al., 1982) from a mixture of poly $\mathrm{A}^{+}$ RNAs prepared from NC4 cells that had developed for 12 and $20 \mathrm{~h}$ as described by Kay et al. (1987). The library was screened under conditions of low stringency with a nick-translated cDNA clone encoding a portion of CABPI (Grant \& Tsang, 1990) as described by Maniatis et al. (1982). To isolate full-length cDNAs, a $\lambda g t 11$ cDNA library, kindly provided by Dr Peter Devreotes (Klein et al., 1988), was screened under high stringency conditions using a nick-translated cDNA isolated from the cDNA library described above.

DNA sequencing. The nucleotide sequences of the cDNAs were determined by the chain termination method (Sanger et al., 1977) using $\left[{ }^{35}\right.$ S]dATP (Amersham) as the radiolabel.

DNA blot hybridization. Genomic DNA was isolated from strain AX2 by centrifugation through $\mathrm{CsCl}$ density gradients (Firtel \& Bonner, 1972), and Southern blotting was performed as described by Maniatis et al. (1982). The filters were prehybridized and hybridized at either $37^{\circ} \mathrm{C}$ (moderate stringency) or $42^{\circ} \mathrm{C}$ (high stringency). They were washed briefly in $2 \times \mathrm{SSC}, 0.1 \% \mathrm{SDS}$ at room temperature and then twice for $30 \mathrm{~min}$ in $2 \times \mathrm{SSC}, 0.1 \%$ SDS at $65^{\circ} \mathrm{C}$ (moderate stringency) or $0.1 \times \mathrm{SSC}, 0.1 \% \mathrm{SDS}$ at $68^{\circ} \mathrm{C}$ (high stringency)

$R N A$ blot hybridization. Five micrograms of poly $\mathrm{A}^{+} \mathrm{RNA}$ isolated from NC4 cells at various times of development was fractionated on $1.5 \%(\mathrm{w} / \mathrm{v})$ agarose formaldehyde gels and transferred to Genescreen membrane (New England Nuclear) (Maniatis et al., 1982). The membrane was probed using the high-stringency conditions employed for DNA blot analysis as described above.

Construction of actin 15-cDNA fusion. Oligonucleotide-directed mutagenesis (Kunkel, 1985) was used to introduce a HindIII site at the translation initiation codon of the clone pDdM34. The presence of the desired mutation was confirmed by DNA sequence analysis (Sanger $e t$ al., 1977). Digestion of the resulting plasmid with HindIII released a fragment containing the entire coding region of the cDNA plus $3^{\prime}$ untranslated sequences. This fragment was then inserted into the expression vector $\mathrm{pB} 10$ Act 15BKH (obtained from $\mathrm{Dr} \mathbf{J}$. Williams, Imperial Cancer Research Fund, Clare Hall Laboratories, U.K.).

Transformation of D. discoideum Plasmid DNA was introduced into strain $\mathrm{AX} 2$ by the calcium phosphate coprecipitation technique (Early \& Williams, 1987). Approximately 150 independent transformants were pooled for analysis.

Hybrid selection, cell-free translation, and immunoprecipitation. These techniques were performed as described previously (Grant \& Tsang, 1990).

Metabolic labelling. Bacterially-grown cells were harvested, washed three times with KKP (20 mM-potassium, phosphate, $\mathrm{pH} 6 \cdot 2)$, and then resuspended to a final density of $5 \times 10^{7}$ cells $\mathrm{ml}^{-1}$ in KKP. One millilitre of this suspension was spread over the surface of a $47 \mathrm{~mm}$ diameter polycarbonate membrane filter (Nuclepore) supported on two layers of filter paper (Whatman no. 1) saturated with KKP. After $3 \mathrm{~h}$ incubation at $22^{\circ} \mathrm{C}$, the filters were transferred to a clean, dry Petri dish and $20 \mu \mathrm{Ci}(740 \mathrm{KBq})\left[{ }^{35} \mathrm{~S}\right]$ methionine was added directly to them. After a $3 \mathrm{~h}$ tabelling period, the cells were washed off the filters, lysed and analysed by immunoprecipitation as described previously (Tsang \& Tasaka, 1986).

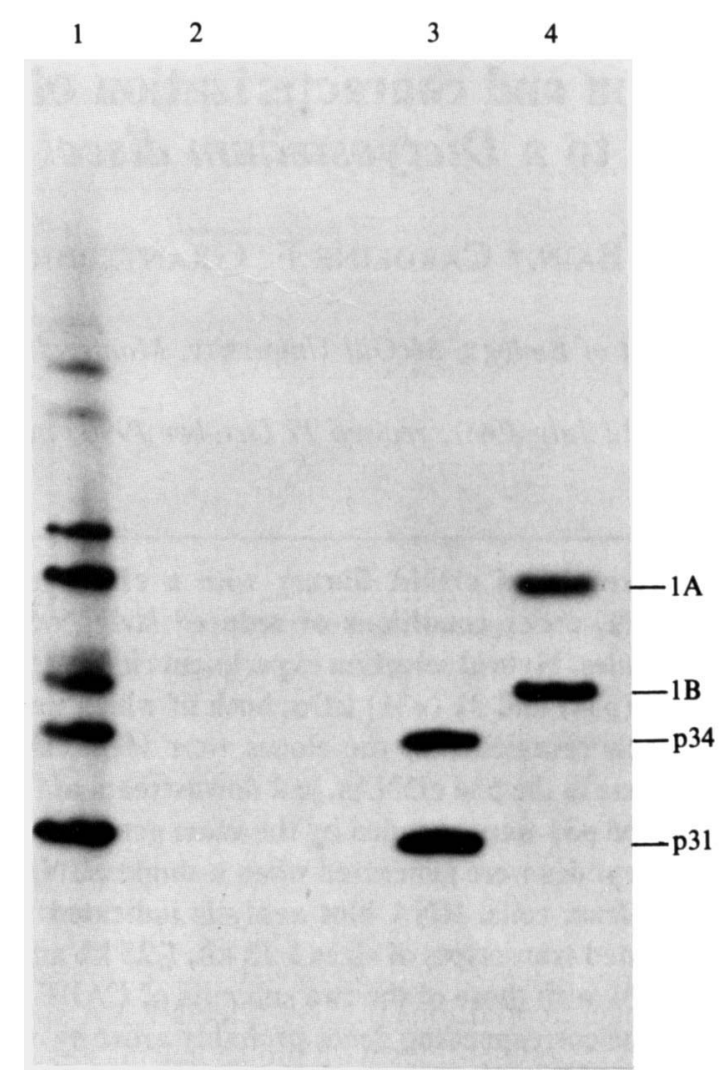

Fig. 1. Identification of pC5D9 by hybrid selection of RNA. PolyA ${ }^{+}$ RNA, isolated from cells that had developed for $17 \mathrm{~h}$, was hybridized under stringent conditions to denatured, filter-bound pC5D9 DNA. After washing the filters to remove the nonspecifically bound material, RNA that had hybridized to PC5D9 was eluted and translated in vitro using a rabbit reticulocyte lysate. The translation products were analysed by immunoprecipitation with an anti-CABP1 monoclonal antibody followed by SDS-PAGE. As a positive control, the CABPI cDNA pC6H6 was also used to select RNA. Lane 1, immunoprecipitated products from the translation of $1 \mathrm{mg}^{\text {polyA }}{ }^{+} \mathrm{RNA}$; lane 2 , no exogenous RNA added to translation reaction; lane 3, immunoprecipitated products from the translation of RNAs complementary to pC5D9; lane 4, immunoprecipitated products from the translation of RNAs complementary to pC6H6. The two subunits of CABP1, $1 \mathrm{~A}$ and $1 \mathrm{~B}$, as well as p34 and p31, are labelled.

\section{Results}

Isolation of $c D N A$ s encoding CABP1-related polypeptides

To isolate clones encoding related polypeptides, we screened a cDNA library with a $0.67 \mathrm{~kb}$ cDNA containing a portion of the CABPl coding sequence (Grant \& Tsang, 1990) under conditions of reduced stringency. Besides CABP1 cDNAs, this approach led to the isolation of a single related cDNA species, termed pC5D9. In order to identify this clone, messenger RNAs complementary to pC5D9 were isolated by hybrid selection, translated in vitro, and analysed by immuno- 

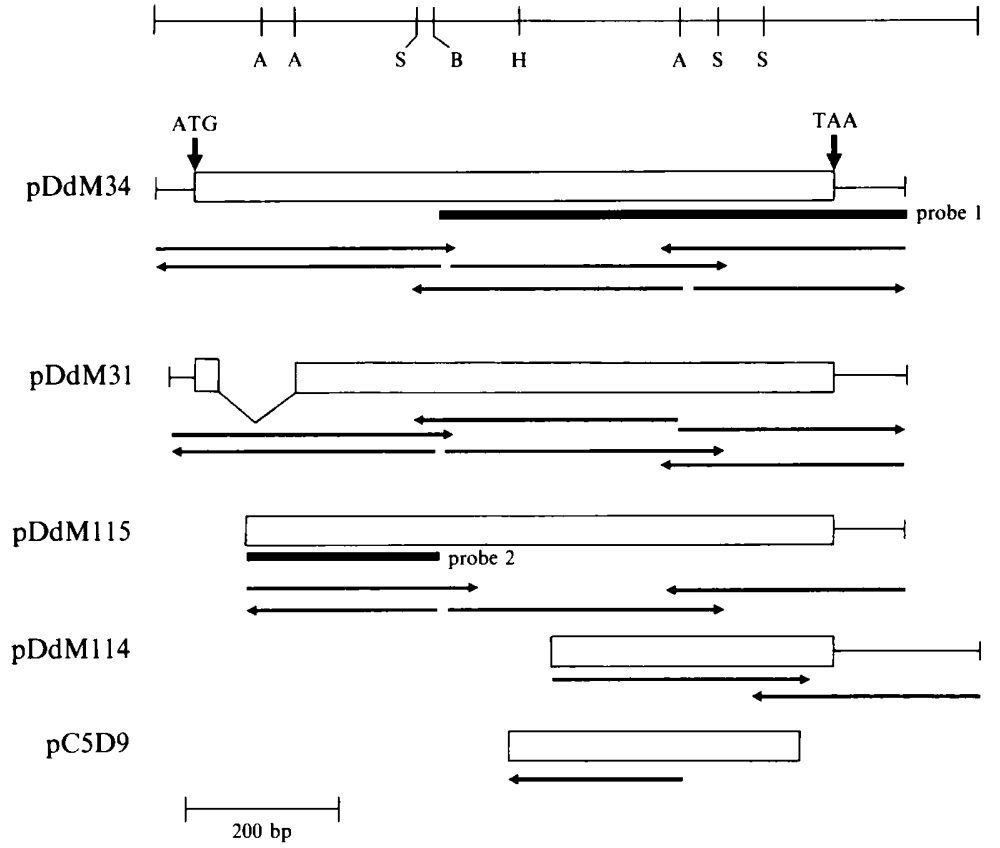

Fig. 2. CABP1-related cDNAs. cDNAs isolated from the phage library were subcloned into the EcoRI site of the plasmid vector Bluescript. The clone isolated from the plasmid library, pC5D9, is contained in the PstI site of pBR322. The open reading frame in each clone is represented by an open rectangle; untranslated sequences are indicated by a line. The restriction fragment used to probe the Southern blot (Fig. 4) is labelled probe 1, while the segment used to probe the Northern blot (Fig. 5) is designated probe 2 . The strategy used to determine the nucleotide sequences of the clones is indicated by the arrows. The restriction map of the cDNA clones is located at the top of the figure. A, AluI; S, Sau3A; B, BamHI; H, HincII. precipitation followed by SDS-PAGE. As Fig. 1 shows, under stringent conditions pC5D9 selects RNA species which encode polypeptides with molecular masses of 34 and $31 \mathrm{kDa}$, both of which are recognized by the antiCABP1 antibody. This observation suggests that pC5D9 contains sequences complementary to the transcripts coding for p34 and p31, and that these two transcripts share substantial similarity at the nucleotide level.

The insert in pC5D9 is only about 400 nucleotides long. To isolate full-length cDNAs for p34 and p31, a dgt11 cDNA library, kindly provided by Dr Peter Devreotes (Klein et al., 1988), was screened under highly stringent conditions using pC5D9 as a probe. Twelve positive clones were obtained after screening approximately 200000 plaques.

\section{Sequence analysis of $c D N A s$}

To facilitate further analysis, the longest cDNAs were subcloned into Bluescript plasmids. Fig. 2 describes the resulting clones and their restriction map as well as the strategy employed to determine their nucleotide sequences.

The two longest cDNAs, pDdM34 and pDdM31, have been sequenced completely on both strands. Each clone contains only a single long open reading frame flanked by $5^{\prime}$ and $3^{\prime}$ untranslated sequences. The nucleotide and deduced amino acid sequences of the cDNAs are illustrated in Fig. 3.

Remarkably, pDdM34 and pDdM31 are completely identical except for the presence of a 102 nucleotide segment located at the $5^{\prime}$ end of $\mathrm{pDdM} 34$, just downstream of the translation initiation codon. This element does not disrupt the open reading frame, thereby leading to the production of a protein containing an extra 34 amino acids compared to the polypeptide encoded by pDdM31.

\section{DNA blot analysis}

Southern analysis was performed using the $3^{\prime}$ BamHIEcoRI fragment common to both pDdM34 and pDdM31 as a probe (probe 1 in Fig. 2). As Fig. 4(a) shows, only a single band hybridized to the probe under conditions of high stringency for all enzymes tested. Even if the stringency of hybridization was reduced, the only additional bands recognized by this probe contain the CABPl gene (Fig. $4 b$ ). Therefore, the transcripts complementary to pDdM34 and pDdM31 appear to be derived from a single gene.

\section{RNA blot analysis}

To examine the pattern of expression of the transcripts complementary to pDdM 34 and $\mathrm{pDdM} 31$, polyA ${ }^{+}$RNA isolated from cells at various times of development was analysed by RNA blotting. Highly stringent conditions were employed to prevent hybridization to CABPl RNAs. As is shown in Fig. 5, three transcripts with lengths of approximately $1.15 \mathrm{~kb}, 1.25 \mathrm{~kb}$ and $1.4 \mathrm{~kb}$ were detected during growth and at all times of development, although their relative amounts were variable. 
*pDdM34 *pDdM31

1 GTATAAAAAAAAAAAAAAAAAAAAAAAAAAAAAAACAAATAAATTAAAGAAA ATG TAT AAT CCA CCA CCA $\begin{array}{lllllll}M & Y & N & P & P & P & 6\end{array}$

71 CCA TCC GGT TCA CAA GGT AAC AAT AAT TAT TAT AGA CAA CCA TCA TCC ACA CCG GGT $\begin{array}{llllllllllllllllllll}\mathbf{P} & \mathrm{S} & \mathrm{G} & \mathrm{S} & \mathrm{Q} & \mathrm{G} & \mathrm{N} & \mathrm{N} & \mathrm{N} & \mathrm{Y} & \mathrm{Y} & \mathbf{R} & \mathbf{Q} & \mathbf{P} & \mathrm{S} & \mathrm{S} & \mathrm{T} & \mathbf{P} & \mathbf{G} & 25\end{array}$ *pDdM115

128 GTT TCT AAC CCA AAT CCA CAA GCT AAT CAA TTT TTA CCA CCT CCA CCT TCT AAT ACA

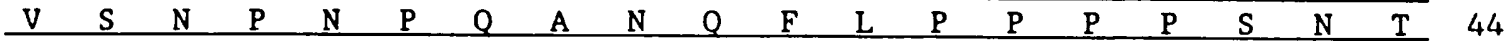

185 CAA GCT CCA AGA CCA GGA TTT CCA CCA AGT GCT CCA CCA CCA AGT GCC CCA GCA GGA $\begin{array}{llllllllllllllllllll}\mathbf{Q} & \mathrm{A} & \mathrm{P} & \mathrm{R} & \mathrm{P} & \mathrm{G} & \mathrm{F} & \mathrm{P} & \mathrm{P} & \mathrm{S} & \mathrm{A} & \mathbf{P} & \mathbf{P} & \mathrm{P} & \mathrm{S} & \mathrm{A} & \mathrm{P} & \mathbf{A} & \mathbf{G} & 63\end{array}$

242 CAA TAC AGT ATG CCA CCA CCA CCA CAA CAA CAG CAA CAA GCA GGA CAA TAT GGT ATG $\begin{array}{llllllllllllllllllll}\mathrm{Q} & \mathrm{Y} & \mathrm{S} & \mathrm{M} & \mathrm{P} & \mathrm{P} & \mathrm{P} & \mathrm{P} & \mathrm{Q} & \mathrm{Q} & \mathrm{Q} & \mathrm{Q} & \mathrm{Q} & \mathrm{A} & \mathrm{G} & \mathrm{Q} & \mathrm{Y} & \mathrm{G} & \mathrm{M} & 82\end{array}$

299 CCA CCA CCA CCA TCA GGT TCA GGT ATA GGT ACA GGT GTT TCA TTA GTA AAA GAT CAA $\begin{array}{llllllllllllllllllll}P & P & P & P & \text { S } & G & \text { S } & \text { G } & \text { I } & \text { G } & \text { T } & \text { G } & \text { V } & \text { S } & \text { L } & \text { V } & \text { K } & \text { D } & \text { Q } & 101\end{array}$

356 CAA ATT TCA TTA AGT AAA GAG GAT CCA TAT CTT AGA AAA TTA ACA GTA GGT TTA GGT $\begin{array}{llllllllllllllllllll}\mathrm{Q} & \mathrm{I} & \mathrm{S} & \mathrm{L} & \mathrm{S} & \mathrm{K} & \mathrm{E} & \mathrm{D} & \mathrm{P} & \mathrm{Y} & \mathrm{L} & \mathrm{R} & \mathrm{K} & \mathrm{L} & \mathrm{T} & \mathrm{V} & \mathrm{G} & \mathrm{L} & \mathrm{G} & 120\end{array}$

413 TGG GAT GTA AAT ACA ACA CCA AGT GCA CCA TTT GAT TTG GAT GCA GTA GTT TTT ATG $\begin{array}{llllllllllllllllllll}W & D & V & N & T & T & P & S & A & P & F & D & \text { L } & \text { D } & \text { A } & \text { V } & \text { V } & \text { F } & \text { M } & 139\end{array}$

470 TTG GGT GCA AAT GGT ATG GTT CGT CAA CCA GCA GAT TTT ATT TTT TAT AAT AAC AAA $\begin{array}{llllllllllllllllllll}\mathrm{L} & \mathrm{G} & \mathrm{A} & \mathrm{N} & \mathrm{G} & \mathrm{M} & \mathrm{V} & \mathrm{R} & \mathrm{Q} & \mathrm{P} & \mathrm{A} & \mathrm{D} & \mathrm{F} & \mathrm{I} & \mathrm{F} & \mathrm{Y} & \mathrm{N} & \mathrm{N} & \mathrm{K} & 158\end{array}$

527 CAA TCT AGG GAT GGT TCA ATC TTT CAT CAT GGT GAT AAT TTA ACA GGT GCA GGT GAT $\begin{array}{llllllllllllllllllll}\mathrm{Q} & \mathrm{S} & \mathrm{R} & \mathrm{D} & \mathrm{G} & \mathrm{S} & \mathrm{I} & \mathrm{F} & \mathrm{H} & \mathrm{H} & \mathrm{G} & \mathrm{D} & \mathrm{N} & \mathrm{L} & \mathrm{T} & \mathrm{G} & \mathrm{A} & \mathrm{G} & \mathrm{D} & 177\end{array}$

584 GGA GAC GAT GAA GTC GTA TCT GTA AAC TTA CAA GCA GTT TCA CCT GAC GTT ACT CGT $\begin{array}{llllllllllllllllllll}\text { G } & \mathrm{D} & \mathrm{D} & \mathrm{E} & \mathrm{V} & \mathrm{V} & \mathrm{S} & \mathrm{V} & \mathrm{N} & \mathrm{L} & \mathrm{Q} & \mathrm{A} & \mathrm{V} & \mathrm{S} & \mathrm{P} & \mathrm{D} & \mathrm{V} & \mathrm{T} & \mathbf{R} & 196\end{array}$

641 TTG GTT TTC GCC GTC ACC ATT CAT CAA CCT GAA TTA AGA AGA CAA AAT TTC GGT ATG $\begin{array}{llllllllllllllllllll}\text { L } & \text { V } & \text { F } & \text { A } & \text { V } & \text { T } & \text { I } & \text { H } & \text { Q } & \text { P } & \text { E } & \text { L } & \text { R } & \text { R } & \text { Q } & \text { N } & \text { F } & \text { G } & \text { M } & 215\end{array}$

698 GTT CCA AGA GCT TTC ATT CGT ATT GCA AAC CAA GAA ACA ACT AGA AAT ATA TGT AGA $\begin{array}{llllllllllllllllllll}\mathrm{V} & \mathrm{P} & \mathrm{R} & \mathrm{A} & \mathrm{F} & \mathrm{I} & \mathrm{R} & \mathrm{I} & \mathrm{A} & \mathrm{N} & \mathrm{Q} & \mathrm{E} & \mathrm{T} & \mathrm{T} & \mathbf{R} & \mathrm{N} & \mathrm{I} & \mathrm{C} & \mathrm{R} & 234\end{array}$

755 TAC GAT CTA ACC AAT GAA GGT GGT ACA AAT ACT GCT ATG ATT GTT GGT GAA GTT TAT $\begin{array}{llllllllllllllllllll}\mathrm{Y} & \mathrm{D} & \mathrm{L} & \mathrm{T} & \mathrm{N} & \mathrm{E} & \mathrm{G} & \mathrm{G} & \mathrm{T} & \mathrm{N} & \mathrm{T} & \mathrm{A} & \mathrm{M} & \mathrm{I} & \mathrm{V} & \mathrm{G} & \mathrm{E} & \mathrm{V} & \mathrm{Y} & 253\end{array}$

812 CGT GAT CCT CAA AAT CCT CAA AAT TGG TCA TTT ATT GCT GTT GGT AAA TCT TTC CCT $\begin{array}{llllllllllllllllllll}R & D & P & Q & N & P & Q & \text { N } & \text { W } & \text { S } & \text { F } & \text { I } & \text { A } & \text { V } & \text { G } & \text { K } & \text { S } & \text { F } & \text { P } & 272\end{array}$

869 GGT GGT TTA CAA TTC CTT TGT CAA ATC TTT GGT GTA AAT GCG TCA TAA ATTTTTTAATT

$$
\begin{array}{lllllllllllllll}
G & G & \text { L } & \text { Q } & \text { F } & \text { L } & \text { C } & \text { Q } & \text { I } & \text { F } & \text { G } & \text { V } & \text { N } & \text { A } & \text { S }
\end{array}
$$

\section{TTAATTTTTTCTTAGGCATTTTTGGCAAATTAGTTGGTTGGAATTTTCACTCATAATTTTGTATACAGATATTTT}

$$
\text { pDdM34* *pDdM31 }
$$

pDdM114*

1003 TTTTTATTCCACATAACACAATAAACAATCACTTTTTTTGTTCTAAAATTAAAAAAAAAAAAAAAAAAAAAAA

Fig. 3. Nucleotide and deduced amino acid sequences of cDNAs. The sequence of the two longest cDNAs, pDdM34 and pDdM31, was determined completely on both strands. The remaining clones have not been sequenced entirely on both strands. The $5^{\prime}$ and $3^{\prime}$ borders of all cDNAs are indicated. The $3^{\prime}$ end of pC5D9 was not determined. The border that is marked represents the $3^{\prime}$ end of our sequencing data for this clone. The sequence which is underlined represents the 102 nucleotide element which is absent from pDdM31. Nucleotide numbers are on the left while amino acid numbers are on the right. 

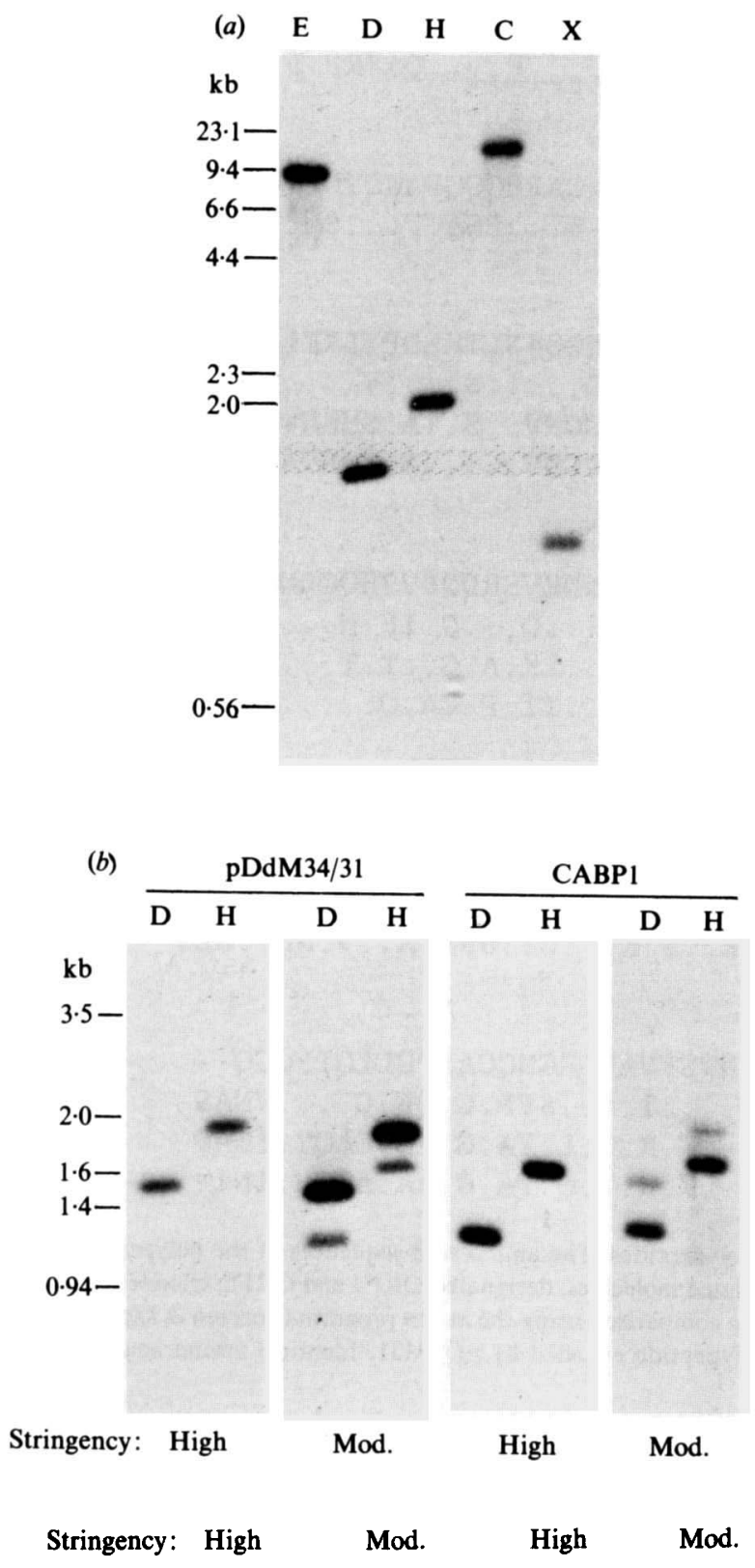

Fig. 4. DNA blot analysis. (a) Genomic DNA digested with a variety of enzymes, size-fractionated on an agarose gel, transferred to a nylon membrane, and then probed under conditions of high stringency with a restriction fragment common to both pDdM34 and pDdM31 (probe 1 in Fig. 2). E, EcoRI; D, DraI ; H, HinfI; C, ClaI; X, XholI. (b) Genomic DNA digested with DraI (D) or Hinf1 (H) and blotted as above. Identical blots were hybridized with either the probe used above or else with a restriction fragment corresponding to the $3^{\prime}$ half of CABP1 under varying degrees of stringency as described in Methods.

\section{Comparison of polypeptides encoded by pDdM34 and pDdM31 to CABPI and related molecules}

As described earlier, the polypeptides encoded by pDdM34 and pDdM31 are identical except for the presence of a 34 amino acid segment inserted near the amino terminus of the larger molecule. Similarly, we

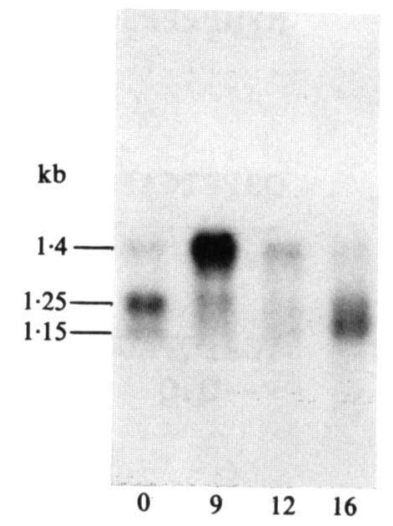

Fig. 5. RNA blot analysis of the expression of RNAs complementary to pDdM34 and pDdM31. PolyA ${ }^{+}$RNA isolated from cells which had developed for $0,9,12$, or $16 \mathrm{~h}$ was fractionated by electrophoresis through an agarose formaldehyde gel and transferred to nitrocellulose. The blot was probed under stringent conditions with a restriction fragment from the cDNA clone pDdM115 (probe 2 in Fig. 2). The sizes of the transcripts which reacted with the probe are indicated.

have found that the two subunits of CABP1 are also identical, except for the presence of an additional 37 amino acids at the amino terminus of CABP1A. We have recently demonstrated that these subunits are the products of a single gene (Grant \& Tsang, 1990), and that they are generated by an alternative splicing mechanism (Grant et al., 1990). An alignment of the deduced amino acid sequences of the polypeptides encoded by pDdM34 and $\mathrm{pDdM} 31$ with those of CABP1A and CABP1B is shown in Fig. 6.

The similarity between these four molecules is remarkable. The amino-terminal sequences are virtually identical, with only two amino acid differences in the first 45 residues. Significantly, the sequence which is specific to the polypeptide encoded by pDdM34 almost exactly matches the region of CABP1 which is found only in CABP1A. Furthermore, the nucleotide sequence encoding this element is flanked precisely by the splice consensus signals GT/AG, suggesting that the transcript which is complementary to pDdM 31 is generated by a splicing process very similar to the one which produces CABP1B (Grant et al., 1990).

Immediately following this highly conserved amino terminus, the polypeptides diverge considerably. This region in CABP1, which is 94 amino acids in length, is very distinctive in its amino acid content. It contains a high proportion of proline and glutamine residues, as well as five copies of the nine amino acid repeat QPAGQYGAP (Grant \& Tsang, 1990). In the molecules encoded by pDdM34 and pDdM31, however, this region contains only 46 residues. Even though it is considerably shorter than the same element in CABP1, its overall character remains very similar since it is also very rich in 


\begin{tabular}{|c|c|}
\hline \multirow{2}{*}{$\begin{array}{l}\text { CABPI } \\
\text { p34/31 }\end{array}$} & MYNPPPPSGSQGNNNYYRQPSSTPGVSNPNPQANQFLPPQPSNTTQTPGGYPPQQQ \\
\hline & 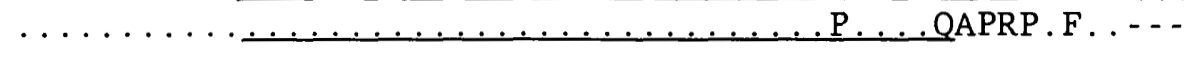 \\
\hline & QRPPTGAPQQPGGYPTPPPPGAPGGYPPQQQPAGQYGAPPQQQPAGQYGAPQPAG \\
\hline & . . . . SM . P . PQ \\
\hline & YGAPQPAGQYGAPQPAGQYGAPPPPPGGAGISLVKNQQISLTKEDPTLRKLTIGL \\
\hline & 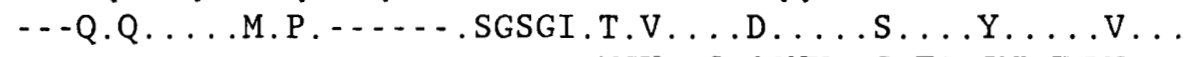 \\
\hline ORF4 & MSV ..S.GGNV ..S.TA.SMKNVLV \\
\hline ORF5 & MAV . . . GGNV . . . . . A. SMNVALV \\
\hline
\end{tabular}

WDVNTTPTAPFDLDAVVFMLNAQGRVRTSQDFIFYNNKVSRDNSVSHQGDNLTGQG

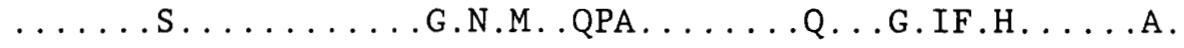
. ARS . DGQD . . . . SA. L.A.N.K. .GDA . . . . . IK.A.G. . T.T . .R. . E.

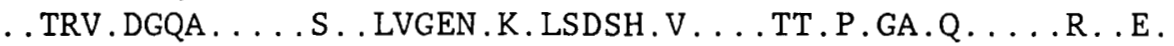
EGDDEVVLVNLQAVSPDVTRLVFAVTIHLADERRQNFTMVPRAFIRVANQETGRNI D . . . . . . . . . . . . . . . QPEL . . . . . . . . . . . . . D. . . SLKIK.D..PG . DKII.V . . . D.QA...S.GQ.SG . . . LV. DDNQTEV D....Q.KID.TK.AA. .KK......E..S.K...G..SNS.M..V.ND . SE .

$$
\begin{aligned}
& \text { CRYDLSQEGGPNTALIAGEVYRDPSNPNNWS FVAVGKGMQGALPGLLQIFGCQ - - }
\end{aligned}
$$

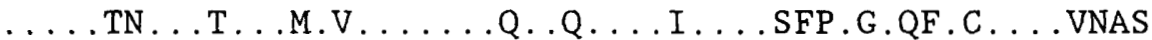

$$
\begin{aligned}
& \text { A. . . TEDASTE. MLF . . L. .HNGA- - .K.R . . L.YA.G.ASVCAQY. INAS } \\
& \text { A.F...EDASTE..M.F. . L. .HGAE- - .K.K...Q.FA.G.AA.ATQH. INI - }
\end{aligned}
$$

Fig. 6. Alignment of the amino acid sequences of CABP1 and related polypeptides. The amino acid sequences of the polypeptides encoded by pDdM34 and pDdM31 were aligned with CABP1 and two related molecules, designated ORF4 and ORF5, encoded by a bacterial plasmid which confers resistance to tellurium anions, by pairwise comparison using the ALIGN program (Pearson \& Lipman, 1988). The underlined sequences are absent from CABP1B and the polypeptide encoded by pDdM31. Identical amino acids are represented by dots, while dashes indicate gaps.

proline and glutamine residues. It also contains two degenerate versions of the nine amino acid repeat found in CABP1, APAGQYSMP and QQAGQYGMP, separated by a polyglutamine tract. However, in these polypeptides, the repeat has been extended to 12 amino acids by the addition of three proline residues to the carboxy terminus. Following this region, the similarity between CABPl and the two related molecules increases again. The carboxy-terminal halves of these polypeptides possess $76 \%$ identity; this value increases to approximately $85 \%$ if conservative changes are included.

We have recently demonstrated that $\mathrm{CABP} 1$ is related to two polypeptides encoded by genes present on a bacterial plasmid which confers resistance to tellurium anions (Grant \& Tsang, 1990). Unsurprisingly, the polypeptides encoded by pDdM34 and pDdM31 also show homology to these bacterial polypeptides (Fig. 6).
No other proteins whose sequences are present in the GenBank, EMBL or NBRF databases have been found to possess significant similarity to these molecules.

\section{Expression of actin 15-pDdM34 fusion gene in Dictyostelium cells}

Southern blot analysis (Fig. 4) suggests that the polypeptides encoded by pDdM34 and pDdM31 are the products of a single gene. To obtain further evidence for this hypothesis, the pDdM34 cDNA clone was expressed in $D$. discoideum cells under the control of the actin 15 promoter using the expression vector pB10Act15BKH. The expression construct codes for a translational fusion in which the amino-terminal 14 amino acids are contributed by vector sequences while the remainder is derived from the open reading frame present in pDdM34 
(a)

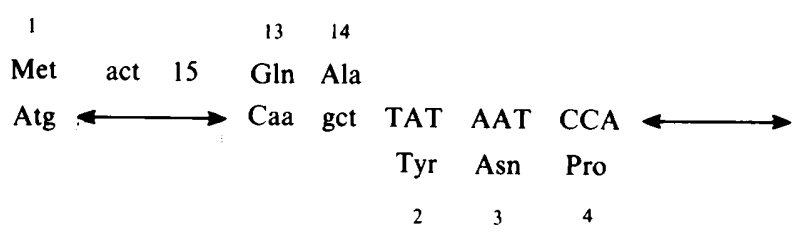

(b)

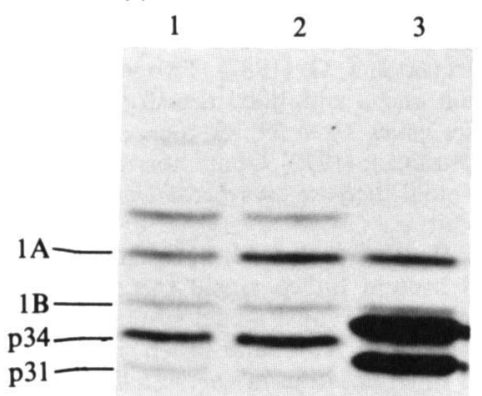

Fig. 7. Expression of pDdM34 under the control of the actin 15 promoter. (a) The details of the actin 15-pDdM34 fusion are shown. The nucleotide sequences provided by the expression vector pB10Act 15BKH are shown in small letters, while those present in pDdM34 are in capitals. The numbers above the sequence represent amino acid position in the fusion, while those below the sequence indicate the codon position in the open reading frame of pDdM34. After introducing the expression construct into $D$. discoideum cells, the transformants were analysed by metabolic labelling with $\left[{ }^{35} S\right]$ methionine followed by immunoprecipitation $(b)$. The two subunits of CABP1, 1A and 1B, are labelled, along with p34 and p31. Lane 1, immunoprecipitated products obtained from control AX2 cells; lane 2, immunoprecipitated products obtained from cells transformed with the expression vector lacking the pDdM34 insert; lane 3, immunoprecipitated products obtained from cells transformed with the actin 15pDdM34 fusion construct.

(Fig. 7a). Stable transformants were isolated and analysed by metabolic labelling with [355]methionine followed by immunoprecipitation with a monoclonal antibody which recognizes CABP1 as well as a number of antigenically related polypeptides (Tsang \& Tasaka, 1986). As Fig. 7(b) clearly shows, transformants carrying this construct express large amounts of two polypeptides which migrate slightly more slowly than the endogenous CABP1-related p34 and p31 molecules. This result indicates that the information required to generate both these polypeptides appears to be present in pDdM34. Furthermore, it strongly supports the hypothesis that these two polypeptides are generated from one gene.

Attempts to use the overexpressing strain for a biochemical analysis of the polypeptides encoded by pDdM34 have not been successful. A more detailed examination of this strain has revealed that the overexpression of these polypeptides is not very stable. In addition, immunofluorescence experiments have demonstrated that the overexpressed molecules appear to be forming aggregates in the cytoplasm and therefore may not be functional (data not shown).

\section{Discussion}

Based on the data presented here, it is very likely that pDdM34 and pDdM31 respectively encode the CABP1related polypeptides $\mathrm{p} 34$ and $\mathrm{p} 31$, which cross-react with anti-CABP1 antibodies and also copurify with CABP1 (Tsang \& Tasaka, 1986). First, hybrid selection analysis of the cDNA clone pC5D9 (Fig. 1) indicates that this sequence is complementary to mRNAs which encode these two molecules. Second, when a cDNA library was screened using this clone as a probe, two different classes of cDNAs were isolated, represented by pDdM34 and pDdM31. Third, the sizes of these two clones agree well with the sizes of two of the transcripts which are detected by Northern blotting (Fig. 5). Finally, these two polypeptides appear to be the products of a single gene (Figs 4 and 7) and are very similar to the two subunits of CABP1, which are also produced from a single gene by alternative splicing (Grant et al., 1990). Taken together, these data strongly suggest that pDdM34 and pDdM31 are complementary to the mRNAs which encode p34 and $\mathrm{p} 31$.

RNA blot analysis demonstrated that three developmentally regulated transcripts with sizes of $1.15 \mathrm{~kb}$, $1.25 \mathrm{~kb}$ and $1.4 \mathrm{~kb}$ are complementary to pDdM34 and pDdM31 (Fig. 5). The 100 bp difference in size between the two smaller transcripts, in conjunction with their overall length, suggests that the cDNA clones are derived from them. The precise origin of the $1.4 \mathrm{~kb}$ mRNA species is unknown at present. We do not believe that this transcript is generated from another closely related gene. Southern analysis demonstrated that only a single gene encodes p34 and p31 (Fig. 4a). Under low stringency conditions, p34/31 probes crossreacted with only one additional gene, that which encodes CABP1 (Fig. $4 b$ ). CABP1 probes, however, do not hybridize to the $1.4 \mathrm{~kb}$ transcript under conditions of high stringency (Grant et al., 1990). Therefore, this mRNA probably corresponds to a third transcript which is generated by the $\mathrm{p} 34 / 31$ gene. The determination of its precise origin and function will require additional analysis.

The three transcripts detected by probes derived from pDdM34 and pDdM31 are present during both growth and development, although their levels are quite variable (Fig. 5). In contrast, p34 and p31 cannot be detected in growing cells by immunoblotting. They appear soon after starvation and then remain at relatively constant levels throughout development (Kay et al., 1987). We do not know the reason for the discrepancy between the expression of the mRNAs and that of the polypeptides. The significance of the fluctuation in transcript levels during development is also not known.

Based on the high similarity between their polypeptide products (Fig. 6), it is probable that the CABP1 and 
p34/31 genes arose by duplication. Subsequent sequence divergence has led to a major change in only one domain, with the remainder of the two genes maintaining close similarity. The virtual identity of the amino termini of p34/31 and CABP1 in contrast to the relatively weaker similarity displayed by the remainder of the proteins suggests that this region of the polypeptides is crucial for function. Furthermore, this area contains the segments which are removed from the CABP1 and p34/31 primary transcripts to generate $\mathrm{CABP} 1 \mathrm{~B}$ and $\mathrm{p} 31$. The strong conservation of this feature between the two genes implies that the ability to generate two polypeptides is also very important.

The high degree of similarity between $\mathrm{p} 34 / 31$ and CABP1 also raises the question of whether these proteins are functionally identical. We do not yet know whether p34 and p31 possess the cAMP binding activity displayed by CABP1. We have previously shown that the developmental regulation and intracellular distribution of these molecules are quite different (Kay et al., 1987). These differences suggest that these molecules do not perform exactly the same functions in the cell.

The functional relationship between p34 and p31 is not clear. Since CABP1 operates as a heterodimer between CABP1A and CABP1B (Tsang \& Tasaka 1986), it is possible that p34 and p31 also associate to form one functional protein. Alternatively, all four polypeptides may be required to form an active complex. More study is required to clarify whether or not this is the case.

The discovery of very high similarity between CABP1, p34/31 and two bacterial polypeptides encoded by a plasmid which confers resistance to tellurium anions (Jobling \& Ritchie, 1988) (Fig. 7) was completely unexpected. This close similarity suggests that these molecules have a common origin. It also implies that they probably have similar functions. Unfortunately, the mechanism which imparts tellurium resistance in bacteria is not known, so we can gain no clues as to the function of $\mathrm{p} 34 / 31$ and $\mathrm{CABP} 1$. The results described in this paper directly demonstrate that the $D$. discoideum cAMP binding protein CABPI belongs to at least a small family of related polypeptides. Additional distinct cDNA clones which encode other molecules which are recognized by anti-CABP1 monoclonal antibodies are currently being analysed in our laboratory. These studies should allow us to determine the precise size of the CABP1 family and may also provide some clues as to the functions of these polypeptides.

We are grateful to Dr P. Devreotes for supplying us with the $\lambda$ gtll library and $\mathrm{Dr} \mathrm{J}$. Williams for providing the expression vector
pB10Act15BKH. This work was supported with grants from NSERC and NCI of Canada.

\section{References}

Early, A. E. \& Williams, J. G. (1987). Two vectors which facilitate gene manipulation and a simplified transformation procedure for Dictyostelium discoideum. Gene 59, 99-106.

FIRTEL, R. A. \& BONNER, J. (1972). Characterization of the genome of the cellular slime mold Dictyostelium discoideum. Journal of Molecular Biology 66, 339-361.

Firtel, R. A., Van HaAstert, P. J. M., Kimmel, A. R. \& Devreotes P. N. (1989). G protein linked signal transduction pathways in development: Dictyostelium as a model system. Cell 58, 235-239.

GERISCH, G. (1987). Cyclic AMP and other signals controlling cell development and differentiation in Dictyostelium. Annual Review of Biochemistry 56, 553-579.

Grant, C. E. \& Tsang, A. (1990). Cloning and characterization of cDNAs encoding a novel cyclic AMP-binding protein in Dictyostelium discoideum. Gene (in the Press).

Grant, C. E., Bain, G. \& Tsang, A. (1990). The molecular basis for the alternative splicing of the CABPI transcripts in Dictyostelium discoideum. Nucleic Acids Research 18, 5457-5463.

Jobling, M. G. \& RitchiE, D. A. (1988). The nucleotide sequence of a plasmid determinant for resistance to tellurium anions. Gene $\mathbf{6 6}$, $245-258$.

KaY, C. A., Noce, T. \& Tsang, A. S. (1987). Translocation of an unusual cAMP receptor to the nucleus during development of Dictyostelium discoideum. Proceedings of the National Academy of Sciences of the United States of America 84, 2322-2326.

KAY, R. R. (1989). Evidence that elevated intracellular cyclic AMP triggers spore maturation in Dictyostelium. Development 105, 753-759.

KIMMEL, A. R. (1987). Different molecular mechanisms for cAMP regulation of gene expression during Dictyostelium development. Developmental Biology 122, 163-171.

Klein, P. S., Sun, T. J., SAXe, C. L., Kimmel, A. R., Johnson, R. L. \& DEVREOTES, P. N. (1988). A chemoattractant receptor controls development in Dictyostelium discoideum. Science 241, 1467-1472.

KuNKeL, T. A. (1985). Rapid and efficient site-specific mutagenesis without phenotypic selection. Proceedings of the National Academy of Sciences of the United States of America 82, 488-492.

LoOmis, W. F. (editor) (1982). The development of Dictyostelium discoideum. New York: Academic Press.

Maniatis, T., Fritsch, E. F. \& SAmbrooK, J (1982). Molecular cloning : a Laboratory Manual. Cold Spring Harbor, NY: Cold Spring Harbor Laboratory.

Pearson, W. R. \& Lipman, D. J. (1988). Improved tools for biological sequence analysis. Proceedings of the National Academy of Sciences of the United States of America 85, 2444-2448.

Riley, B. B., Jensen, B. R. \& BarClay, S. L. (1989). Conditions that elevate intracellular cAMP levels promote spore formation in Dictyostelium. Differentiation 41, 5-13.

SANGer, F., Nicklen, S. \& Coulson, A. R. (1977). DNA sequencing with chain-terminating inhibitors. Proceedings of the National Academy of Sciences of the United States of America 74, 54635467.

Tsang, A. S., Grant, C., Kay, C., Bain, G., Greenwood, M., Noce, T. \& TASAKA, M. (1988). Characterization of an unusual cAMP receptor and its related polypeptides in Dictyostelium discoideum. Developmental Genetics 9, 237-245.

TSANG, A. S. \& TASAKA, M. (1986). Identification of multiple cyclic AMP binding proteins in developing Dictyostelium discoideum cells. Journal of Biological Chemistry 261, 10753-10759. 